\title{
OTIONOMTES
}

Revista de economía, empresa y sociedad

Dossier «Revolución 4.0: ¿progreso o precarización?»

Coordinador: Josep Lladós

PROCESOS DE GENERACIÓN DE VALOR

\section{Industria 4.0 y resultados empresariales en Fspaña: un primer escaneado}

\section{Joan Torrent-Sellens}

Catedrático de economía de los Estudios de Economía y Empresa (UOC)

\begin{abstract}
RESUMEN Este artículo analiza la relación entre los usos de las tecnologías de la industria 4.0 (14.0), la generación de valor y los resultados empresariales. Mediante una muestra de 1.525 empresas industriales españolas en 2014, se identifican los usos de cuatro tecnologías básicas de la 14.0: 1) diseño industrial asistido por computadora, CAD; 2) robótica; 3) sistemas flexibles de producción; y 4) hardware y software de control numérico de la actividad-, se construye un indicador aditivo y se estudia la asociación estadística con la generación de valor y los resultados de la empresa. La investigación ha obtenido tres resultados principales. En primer lugar, cabe destacar su estado incipiente; un $72,5 \%$ de las empresas industriales españolas o bien no utiliza, o bien utiliza muy moderadamente las tecnologías identificadas de la 14.0. Sin embargo, y en segundo lugar, debe señalarse que los usos de estas tecnologías se asocian a un proceso de generación de valor de la empresa industrial más intensivo en I+D y en capital humano, más innovador, más digital y más sostenible. En tercer lugar, la investigación también concluye que las empresas con unos usos más intensivos de las tecnologías 14.0 presentan mejores resultados en términos de ventas, valor añadido, exportaciones y margen bruto de explotación. Especialmente relevantes son los resultados de la productividad y del empleo. Las empresas industriales intensivas en usos de las tecnologías 14.0 son un $30 \%$ más eficientes que las empresas que no usan estas tecnologías. También son capaces de ocupar a un número mucho mayor de empleados (el doble de la media industrial) y de retribuirlos mucho mejor (12,4\% por encima de la media industrial). Por último, en el artículo también se discute el papel que la 14.0 podría tener como nueva tecnología de propósito general.
\end{abstract}

PALABRAS CLAVE Industria 4.0; digitalización; robótica; manufactura inteligente; empresa industrial; productividad; España.

\section{Industry 4.0 and firm performance in Spain: a first scan}

ABSTRACT This article analyses the relationship between the uses of Industry 4.0 technologies (14.0), the value generation and firm results. Based on a sample of 1,525 Spanish industrial firms for 2014, the uses of four basic 14.0 technologies are identified: 1) computer-aided industrial design (CAD); 2) robotics; 3) flexible production systems; and 4) the activity's numerical control machinery 
and software, an additional indicator is constructed and the statistical association with the value generation and firm results are studied. The research has obtained three main results. First of all, it is worth noting its incipience. $72.5 \%$ of Spanish industrial firms either do not use or use very moderately the 14.0 technologies. Despite of this and secondly, it should be noted that the uses of these technologies are associated with a value generating process in industrial firms which is more intensive in R\&D and human capital, more innovative, more digital and more sustainable. And, thirdly, the research also concludes that firms with more intensive uses of 14.0 technologies have better results in terms of sales, value added, exports and gross operating margin. Productivity and employment results are especially relevant. 14.0 intensive industrial firms are 30\% more efficient than firms that do not use these technologies. They are also able to take on a much larger number of employees (twice the industrial average) and to pay them much better (12.4\% above the industrial average). Finally, the article also discusses the role that 14.0 could play as a new general purpose technology.

KEYWORDS industry 4.0; digitisation, robotics; smart manufacturing; industrial firm; productivity; Spain

\section{Introducción}

Habitualmente, desde la economía entendemos la tecnología como el fondo social de conocimiento sobre las artes industriales. Es decir, todo el conjunto de saberes, no únicamente los científicos y los tecnológicos (saber-qué y saber-por qué) y particularmente las habilidades de los agentes económicos y de las organizaciones (saber-cómo y saber-quién), que inciden sobre la actividad económica. Por consiguiente, nos aproximamos a la tecnología a partir del conocimiento que genera todo conjunto de instrumentos, máquinas o técnicas para la acción instrumental (Torrent-Sellens, 2004). Las tecnologías de propósito o de utilidad general (general purpose technologies, GPT) son familias de saber aplicado de orden superior, en el sentido que derivan en aplicaciones tecnológicas más específicas y de orden inferior. Por ejemplo, las tecnologías vinculadas con la máquina de vapor, la electricidad, el motor de combustión interna o el ordenador se consideran tecnologías de utilidad general, porque mediante su capacidad de conexión (plataforma) con otras tecnologías configuran procesos de convergencia tecnológica, innovaciones derivadas, complementariedades con otros activos económicos, como por ejemplo con la inversión en intangibles y, finalmente, nuevos modelos de negocio, nuevas fuentes de eficiencia y nuevas palancas de crecimiento económico (Bodrozic y Adler, 2018).

A partir de la investigación sobre las revoluciones industriales, es decir, el conjunto de cambios disruptivos en la tecnología (tecnologías de utilidad general) y en la estructura económica (paradigmas técnico-económicos o ciclos económicos de larga duración) que se interconectan con cambios sociales y culturales de primer orden, los economistas hemos aprendido una lección significativa. En cada una de las tres revoluciones industriales que se han evidenciado hasta el momento, un factor o un conjunto de factores productivos se consolidan como fuentes del crecimiento económico, del cambio competencial del empleo y de la estructura social. Estos factores nunca son la tecnología sobre la que se sustenta el cambio económico. Por ejemplo, en la Primera Revolución Industrial, la irrupción de la máquina de vapor consolidó el proceso de substitución de instrumentos por máquinas, la aparición del trabajo fabril y de los trabajadores industriales urbanos. En la Segunda Revolución Industrial, la electricidad y el motor de combustión interna incentivaron la incorporación del conocimiento científico en la industria, la organización científica, la atomización y la alineación del trabajo, así como los métodos de producción en masa. En la Tercera Revolución Industrial, los avances de las tecnologías de la información y la comunicación (TIC), del internet no interactivo y del comercio electrónico configuraron la era de la información y del conocimiento. En los tres casos, la tecnología determinó mejoras de eficiencia (productividad total de los factores) e, incluso, la aparición de nuevos sectores de actividad. Pero, el efecto multiplicador, la generación de nuevos factores de pro- 
ductividad, se alcanza cuando los bienes y los servicios generados por la nueva oleada tecnológica son utilizados por el resto de actividades económicas e interaccionan con los factores productivos, los modelos de negocio, las estructuras de mercado y la organización de la economía.

Durante los últimos años, y a partir de las transformaciones vinculadas con la Tercera Revolución Industrial, una nueva oleada de cambio tecnológico digital y disruptivo ha vuelto a generar transformaciones de calado en el comportamiento, la estructura y los resultados de los agentes económicos, los modelos de negocio y los mercados (Trajtenberg, 2018). La robótica, la inteligencia artificial, el aprendizaje de las máquinas, el aprendizaje profundo, la computación en la nube, los grandes datos, la impresión 3D, el internet de las cosas, los medios y las redes de comunicación social y las plataformas colaborativas, entre otras, parece que se configurarán como una nueva base tecnológica convergente de propósito general y que definirán nuevas fuentes agregadas de productividad y de crecimiento económico (Torrent-Sellens y Díaz-Chao, 2018). A las puertas de la Cuarta Revolución Industrial, la nueva oleada tecnológica también impulsa transformaciones de calado en la actividad industrial, que se revisaran a continuación.

\section{Industria 4.0: definición, componentes e implicaciones}

La industria 4.0 (en adelante 14.0) es un constructo multidimensional y en constante evolución utilizado para definir el actual proceso de transformación digital en los sistemas manufactureros de producción, que evolucionan hacia procesos más flexibles y hacia una toma de decisiones estratégica y operativa basada en el análisis de datos masivos en tiempo real (Porter y Heppelmann, 2014; Xu et al., 2014). La 14.0 tiene una base tecnológica que interacciona con los sistemas de producción y de organización del trabajo. En función de la relevancia de estas dimensiones, la investigación económica ha realizado diversas aproximaciones. Desde el punto de vista tecnológico, se ha señalado que la 14.0 integra elementos físicos tradicionales (como máquinas o dispositivos de producción) y elementos digitales (como sensores y software en red) con el objetivo de generar datos que permitan una gestión empresarial más eficiente. De hecho, estas complementariedades entre los entornos físicos y virtuales sobrepasan el ámbito tecnológico y se extienden hacia el conjunto de elementos de valor y las fuerzas competitivas de la actividad industrial. En este sentido, otras visiones se aproximan a la 14.0 como un modelo de organización y de gestión de la cadena de valor durante el ciclo de vida de los productos o, incluso, como un concepto colectivo que aglutina nuevas tecnologías digitales y nuevas formas de organización de la cadena de valor. Considerando sus complementariedades tecnológicas, estratégicas, organizativas y de producción, la 14.0 se puede interpretar como «un proceso de fabricación integrado, adaptado, optimizado, orientado al servicio e interoperable que se correlaciona con algoritmos, grandes datos y tecnologías elevadas» (Lu, 2017, pág. 3).

La 14.0 tiene un fundamento, una base material, tecnológica. Se basa en la utilización de las tecnologías digitales, especialmente las de segunda oleada, como el internet de las cosas (Internet of Things, loT), internet de los servicios (Internet of Services, loS), la computación en la nube (cloud computing), las redes inalámbricas de sensores, o los grandes datos (big data) para recopilar datos en tiempo real y analizarlos con el objetivo de generar información útil y mejorar la eficiencia de los sistemas de fabricación (Wang et al., 2016). Esta recopilación y análisis de datos masivos permite la creación de sistemas ciberfísicos (cyber-physical system, CPS) que consolidan la tendencia hacia la terciarización de la industria (services to manufacturing) y que evolucionan tecnológicamente a los sistemas integrados de producción. Los CPS son «sistemas de entidades computacionales colaborativos que están en conexión intensiva con el entorno físico inmediato y sus procesos de producción, y que, al mismo tiempo, proporcionan y utilizan servicios de acceso y procesamiento de datos disponibles en Internet» (Monostori et al., 2016). Por ejemplo, los controladores de sensores o máquinas de control numérico que intercambian datos masivos mediante terminales informáticos integrados, aplicaciones inalámbricas o computación en la nube. Con los sistemas CPS, las empresas pueden: 1) hacer converger sus entornos físicos y virtuales; 2) mejorar la planificación, análisis, modelización, diseño, implantación y mantenimiento del proceso de fabricación; y 3) aumentar la productividad, fomentar el crecimiento, modificar el rendimiento de la fuerza de trabajo, y producir bienes de más calidad con menos costes por medio de la recopilación y el análisis masivo de datos. 
Como resultado de que los CPS: 1) combinan datos e información con productos y factores físicos de producción; 2) monitorizan y crean una copia virtual del mundo físico; y 3) integran la fábrica con todo el ciclo de vida del producto y con las actividades de las cadenas de suministro, las implicaciones para el cambio en las formas de organización del trabajo son más que evidentes. Las posibilidades para la toma autónoma y descentralizada de decisiones, la comunicación y la cooperación entre las tecnologías de la automatización y las personas en tiempo real, y la creciente transición desde los productos hacia los servicios por parte de todos los agentes que participan en las redes de creación de valor demandan nuevas maneras de organizar el trabajo. La 14.0 también implica importantes modificaciones en el rol que desarrollan las personas dentro de los sistemas de producción. Las tareas en las nuevas redes de valor se realizan con enfoques de trabajo inteligente (smart work) (Longo et al., 2017). En este sentido, las formas tecnológicas y el trabajo inteligente de la 14.0 reconfiguran los sistemas integrados de producción, que también evolucionan y encajan con la idea de la manufactura avanzada o de la fábrica inteligente (smart manufacturing): un nuevo sistema adaptable donde las líneas flexibles ajustan automáticamente los procesos de producción para múltiples tipos de productos y para condiciones cambiantes, lo que mejora la calidad, la productividad y la flexibilidad, al mismo tiempo que ayuda a conseguir productos personalizados a gran escala y de manera más sostenible con menos consumo de recursos (Dalenogare et al., 2018; De SousaJabbour et al., 2018).

Como no podría ser de otra manera, la utilización de tecnologías, trabajo y producción inteligente acaba por configurar una última dimensión de la 14.0: la de los productos inteligentes (smart products). Mediante el uso combinado de las tecnologías y de los métodos de producción y trabajo 4.0, estos pueden proporcionar información sobre el desarrollo de nuevos productos/servicios, nuevas soluciones para los clientes o nuevas oportunidades para los proveedores de servicios (Porter y Heppelmann, 2015). Del mismo modo, la integración inteligente de toda la cadena de valor (smart supply chain), desde los suministros hasta los distribuidores y los clientes finales, permite a las empresas de la 14.0 la combinación de recursos y la fabricación colaborativa en el sentido de compartir recursos en plataformas industriales, centrarse en sus competencias básicas y desarrollar productos/ servicios complementarios con más valor añadido (Zhong et al., 2017; Tao et al., 2018).

Por último, también cabe destacar la dimensión política de la 14.0. La idea de la 14.0 fue presentada por primera vez en la Feria de Hannover en 2011. En 2013 se convirtió en una iniciativa estratégica del gobierno alemán (Industrie 4.0) que, en colaboración con universidades y empresas, desarrolla un plan de sistemas avanzados de producción (High-Tech Strategy 2020), con el objetivo de aumentar la productividad, la eficiencia y la sostenibilidad de la industria nacional (Kagermann et al., 2013). Muy pronto, planes similares también han sido desarrollados en otros países, como las iniciativas Advanced Manufacturing Partnership en EE. UU., Made in China 2025 en China, la Nouvelle France Industrielle en Francia, o Rumo à Indústria 4.0 en Brasil. Todavía es pronto para una evaluación efectiva de estos programas, pero dan una clara señal de la importancia estratégica que la política pública confiere a la 14.0. Todos estos programas tienen como propósito incentivar los usos de las tecnologías y los sistemas de producción y trabajo 4.0 como punto de partida para la transformación y el renacimiento industrial de sus economías respectivas. El objetivo que se busca es claro: recuperar el peso de la industria como motor económico, y como fuente de eficiencia y creadora de puestos de trabajo de calidad elevada.

\section{Tecnologías 14.0 y resultados empresariales: estado del arte}

Acabamos de constatar que la 14.0 se puede identificar por medio de cuatro pilares fundamentales: las tecnologías de la segunda oleada digital, las redes de valor y la manufactura inteligente, el trabajo inteligente y los productos inteligentes (Frank et al., 2019). A partir de estos cuatro pilares, a continuación se evaluará la relación entre algunos usos de la 14.0 y los resultados de la empresa industrial en España. Como punto de partida, cabe destacar que la investigación al respecto es más bien escasa. Ya hemos señalado que las tecnologías de la 14.0 son capaces de generar una amplia gama de beneficios para la industria, que van desde la fabricación aditiva, la producción flexible y los productos personalizados (Brettel et al., 2014; Weller et al., 2015), hasta el apoyo y la 
adaptación constante de la toma de decisiones (Schuh et al., 2017), la eficiencia en la gestión de los recursos, especialmente los energéticos (Jeschke et al., 2017), o los nuevos modelos de negocio más colaborativos y derivados de la integración horizontal y de las redes de colaboración (Wei et al., 2017).

Sin embargo, gran parte de la evidencia disponible está más relacionada con la investigación sobre cómo las tecnologías de la 14.0 modifican el proceso empresarial de generación de valor que con el estudio de los efectos sobre los resultados de las empresas. Básicamente, esto es así por dos razones. En primer lugar, por la falta de información estadística completa sobre los usos empresariales del conjunto de las tecnologías 14.0; y, en segundo lugar, porque, como ya se ha demostrado en otras oleadas del cambio tecnológico digital, el análisis de los efectos de las tecnologías 14.0 sobre los resultados empresariales también debe tener en cuenta las relaciones de complementariedad que se establecen con otros activos de la empresa, especialmente con el capital humano y con las estructuras de organización del trabajo (Díaz-Chao et al., 2015). En otras palabras, un análisis completo de los efectos de la 14.0 sobre los resultados empresariales debería identificar y considerar sus cuatro pilares: tecnología, manufactura y redes, trabajo y productos inteligentes.

Desde esta perspectiva, una investigación pionera (Müller et al., 2018), que ha utilizado una muestra internacional de 814 empresas que han usado tecnologías de big data y de análisis masivo de datos en el período 2008-2014, obtiene que los usos de estas tecnologías se asocian con mejoras de productividad situadas entre un $3 \%$ y un $7 \%$. Al mismo tiempo, también se concluye que la intensidad tecnológica y la capacidad competitiva del subsector de actividad industrial refuerzan la capacidad de las empresas para mejorar su productividad mediante los activos vinculados con las tecnologías 14.0. De hecho, fuera de los sectores intensivos en tecnología, o con una elevada presión competitiva, los efectos de las tecnologías de big data y de análisis masivo de datos sobre la productividad no son significativos.

Ampliando el número de tecnologías 4.0 y el alcance de sus resultados, otra investigación reciente (Dalenogare et al., 2018) contrastada en una amplia muestra de 2.225 empresas industriales en Brasil durante el año 2016, obtiene datos valorativos, identifica los usos para un conjunto de nueve tecnologías: 1) diseño y manufactura asistidos por computadora (CAD/CAM); 2) sistemas integrados de ingeniería; 3) automatización digital, loT y sensores; 4) líneas de manufactura flexible; 5) sistemas digitales de control de la producción, tipo ERP o MES (Manufacturing Execution System); 6) grandes datos (big data); 7) productos/servicios digitales; 8) manufactura aditiva y 3D; y 9) servicios de computación en la nube (cloud) - y obtiene tres factores de beneficios esperados: 1) para los productos: personalización, calidad y reducción de los tiempos de lanzamiento; 2) para las operaciones: costes operativos, productividad, y visualización y control; y 3) efectos colaterales o secundarios: sostenibilidad y satisfacción de los trabajadores. Sin embargo, los resultados del análisis predictivo son mixtos. Si nos referimos a los beneficios operativos, los sistemas CAD/CAM, la automatización digital y el big data predicen efectos operativos positivos, mientras que la manufactura aditiva predice efectos negativos. El resto de tecnologías 14.0 no predice beneficios operativos esperados.

En España, la Encuesta sobre el uso de TIC y comercio electrónico en las empresas, elaborada por el INE, proporciona información estadística representativa sobre algunos de los usos de las tecnologías 14.0 (figura 1). A principios de 2018, ninguna de las tecnologías de la 14.0 identificadas llegaba a ser utilizada por más de una quinta parte del tejido industrial, configurado por empresas de más de diez trabajadores. Únicamente la robótica $(24,1 \%)$, los sistemas digitales de gestión de la actividad (ERP o CRM mediante programas de software libre) $(22,7 \%)$ y los usos (compras de programas) de computación en la nube (18,7\%) se acercaban a este registro. En cambio, el análisis de big data (7,9\% de empresas industriales), los servicios web de personalización de productos inteligentes por parte de los clientes $(6,5 \%)$ y la impresión 3D $(6,4 \%)$ tenían una presencia minoritaria entre las empresas industriales españolas. 
Figura 1. Usos de las tecnologías 14.0 en España. 2015-2018 (porcentajes de empresas industriales de más de diez trabajadores que usan tecnologías 14.0. Datos del primer trimestre de cada año)

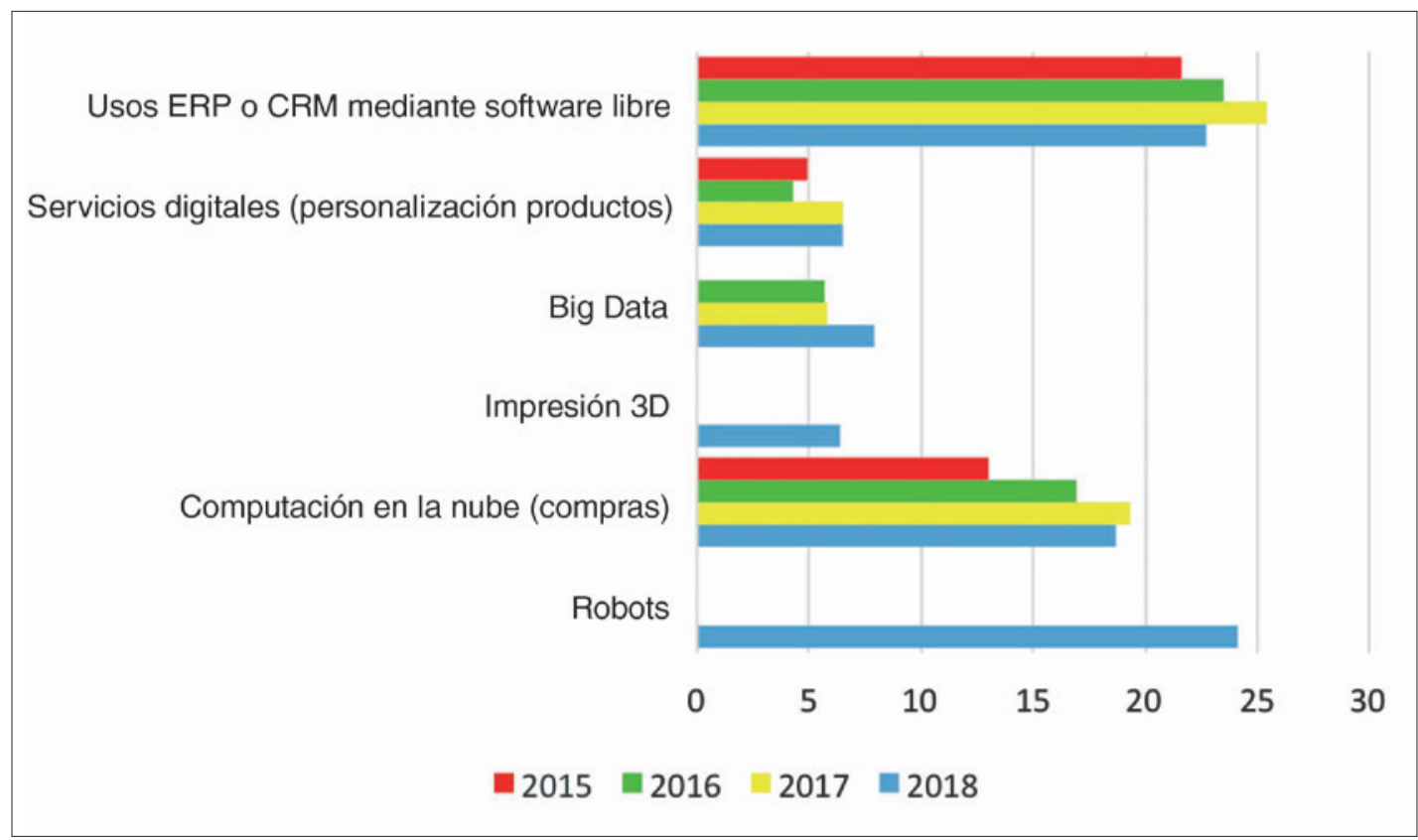

Fuente: Elaboración propia a partir de los datos del INE.

\section{Intensidad de uso en tecnologías 14.0 y resultados empresariales en España: primera evidencia}

Más allá de su valor descriptivo, los datos anteriores no nos permiten estudiar la relación entre los usos de estas tecnologías y los resultados de la empresa industrial en España. Con la intención de aportar una primera evidencia al respecto, se utilizará una fuente de información alternativa: la Encuesta sobre Estrategias Empresariales (ESEE). La ESEE es una encuesta anual, realizada sobre una muestra de unas 1.800 empresas industriales españolas, que elabora la Fundación SEPI, integrada en el Ministerio de Hacienda del Gobierno de España. El cuestionario proporciona información detallada sobre las empresas industriales, especialmente para los ámbitos de la toma estratégica de decisiones (precios, costes, mercados e inversiones) y de la generación interna de valor (estructura empresarial, capital humano, organización, innovación, I+D y usos TIC). Además, también aporta información económica y financiera de los principales indicadores y ratios del balance de situación, así como la cuenta de pérdidas y de ganancias. La encuesta provee datos anuales para el período 1990-2016 (último año disponible) y segmenta la información aportada por dimensión: empresas grandes (más de 200 trabajadores) y pymes (empresas de 10 a 200 trabajadores), y para veinte subsectores de actividad industrial. En este sentido, cabe señalar que se trata de una operación estadística que aporta información representativa y de largo plazo sobre la estrategia, la generación de valor y los resultados económicos y financieros de la empresa industrial, lo que le confiere una gran utilidad para el análisis del conjunto de factores explicativos de la dinámica industrial (Torrent-Sellens, 2018).

Antes de la presentación de los resultados obtenidos, es necesario hacer algunas consideraciones. En primer lugar, la investigación contempla una muestra de 1.525 empresas industriales españolas en el año 2014. En segundo lugar, el análisis se realiza para 2014 porque la información sobre las tecnologías analizadas se obtiene cada cuatro años, y 2014 es el último año con datos disponibles. En tercer lugar, cabe 
señalar que en este primer escaneado se presentará un análisis de comparación de medias (crosstabs) entre un indicador aditivo de tecnologías 14.0 y algunos de los principales resultados de la empresa industrial. El objetivo es determinar la asociación estadística entre este indicador y los resultados empresariales, lo que no designa necesariamente capacidad predictiva, pero sí infiere vinculación estadística, además de aportar un valor descriptivo para los indicadores analizados. Por último, y en cuarto lugar, con el objetivo de proporcionar información sobre la intensidad de uso yendo más allá de los valores dicotómicos que proporcionan los datos iniciales, cabe indicar que se ha construido un indicador aditivo sobre los usos de cuatro tecnologías 14.0: 1) usos de CAD; 2) usos de robótica; 3) usos de sistemas flexibles de producción; y 4) usos de hardware y software de control numérico de la actividad. Estas cuatro variables de entrada tomaban dos valores: 0, no utilización, y 1, utilización. En este sentido, la construcción del indicador aditivo nos determina una variable discreta que toma cinco valores ( 0 a 4). Con el objetivo de hacer más inteligibles los resultados obtenidos, este indicador inicial ha sido recodificado en tres valores: 0, no utiliza ninguna tecnología 14.0; 1, utilización baja: usos de 1 o 2 tecnologías 14.0; 2, utilización intensiva: usos de 3 o 4 tecnologías 14.0. Las frecuencias obtenidas nos determinan que un $29,0 \%$ de las empresas industriales de la muestra no utiliza ninguna tecnología 14.0, que un 43,5\% realiza una utilización baja (usa 1 o 2 tecnologías 14.0), y que el 27,5\% restante de empresas lleva a cabo una utilización intensiva (usa 3 o 4 tecnologías 14.0). Aunque hasta la edición de la ESEE de 2018 no se dispondrá de más información sobre otras tecnologías más propias de la 14.0, como cloud computing, big data o loT, las frecuencias obtenidas nos señalan una utilización media-baja de las tecnologías 14.0: un 72,5\% de las empresas industriales en España o bien no utiliza, o bien utiliza muy modestamente las tecnologías identificadas de la 14.0. 


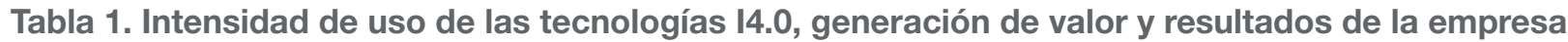
industrial en España. 2014

\begin{tabular}{|c|c|c|c|c|}
\hline Variable/indicador & No 14.0 & Uso bajo & Uso intensivo & Total \\
\hline $\begin{array}{l}\text { Resultados de la empresa } \\
\text { Ventas (miles de euros) } \\
\text { Valor añadido (miles de euros) } \\
\text { Exportaciones (miles de euros) } \\
\text { Margen bruto de explotación (\%) }\end{array}$ & $\begin{array}{r}26.783 \\
4.934 \\
14.069 \\
4,1\end{array}$ & $\begin{array}{r}49.073 \\
11.116 \\
19.494 \\
4,3\end{array}$ & $\begin{array}{r}150.799 \\
28.416 \\
91.687 \\
7,2\end{array}$ & $\begin{array}{r}70.628^{\star \star \star} \\
14.089^{\star \star \star} \\
37.781^{\star \star \star} \\
5,1^{\star}\end{array}$ \\
\hline $\begin{array}{l}\text { Generación de valor } \\
\text { Gasto I+D (miles de euros) } \\
\text { Empleados I+D (\% sobre total empleados) } \\
\text { Gasto externo formación por trabajador (€) } \\
\text { Inversión medioambiental (\% empresas) } \\
\text { Gasto medioambiental (\% empresas) }\end{array}$ & $\begin{array}{r}147,1 \\
1,0 \\
73,5 \\
3,5 \\
10,6\end{array}$ & $\begin{array}{r}661,0 \\
5,4 \\
96,4 \\
9,3 \\
\mathbf{2 6 , 7}\end{array}$ & $\begin{array}{r}2.121,3 \\
12,3 \\
139,2 \\
\mathbf{1 0 , 4} \\
\mathbf{2 1 , 0}\end{array}$ & $\begin{array}{r}910,9^{\star \star \star} \\
6,0^{* \star} \\
101,6^{* \star \star} \\
23,3^{\star \star *} \\
58,3^{\star \star *}\end{array}$ \\
\hline $\begin{array}{l}\text { Tecnología (\% empresas) } \\
\text { Dirección o comité tecnología (\% empresas) } \\
\text { Asesores tecnológicos (\% empresas) } \\
\text { Evaluación tecnologías alternativas (\% empresas) } \\
\text { Serv. programación informática (\% empresas) }\end{array}$ & $\begin{array}{r}3,5 \\
3,0 \\
3,1 \\
23,0\end{array}$ & $\begin{array}{r}9,8 \\
7,5 \\
9,7 \\
38,9\end{array}$ & $\begin{array}{r}9,9 \\
8,3 \\
10,6 \\
26,4\end{array}$ & $\begin{array}{l}23,1^{\star \star \star} \\
18,8^{\star \star \star} \\
23,3^{\star \star \star} \\
88,3^{\star \star \star}\end{array}$ \\
\hline $\begin{array}{l}\text { Innovación (\% empresas) } \\
\text { Innovación producto (\% empresas) } \\
\text { Innovación proceso (\% empresas) } \\
\text { Innovación organizativa (\% empresas) } \\
\text { Innovación en comercialización (\% empresas) }\end{array}$ & $\begin{array}{l}3,0 \\
6,2 \\
3,9 \\
4,7\end{array}$ & $\begin{array}{r}7,5 \\
14,6 \\
8,9 \\
8,6\end{array}$ & $\begin{array}{r}6,1 \\
13,9 \\
8,1 \\
6,2\end{array}$ & $\begin{array}{r}16,5^{\star \star \star} \\
34,7^{\star \star \star} \\
20,8^{\star \star \star} \\
19,4^{\star \star}\end{array}$ \\
\hline $\begin{array}{l}\text { Digitalización (\% empresas) } \\
\text { Compras digitales proveedores (\% empresas) } \\
\text { Ventas digitales empresas (\% empresas) }\end{array}$ & $\begin{array}{l}8,1 \\
2,4\end{array}$ & $\begin{array}{r}17,0 \\
4,2\end{array}$ & $\begin{array}{r}14,4 \\
3,9\end{array}$ & $\begin{array}{r}39,5^{\star \star \star} \\
10,5^{\star \star}\end{array}$ \\
\hline $\begin{array}{l}\text { Productividad y empleo } \\
\text { Productividad (miles de euros por trabajador) } \\
\text { Productividad (euros por hora trabajada) } \\
\text { Empleados (número) } \\
\text { Costes laborales por empleado (euros) }\end{array}$ & $\begin{array}{r}44,1 \\
25,2 \\
71,3 \\
30.736\end{array}$ & $\begin{array}{r}58,8 \\
33,8 \\
158,4 \\
36.439\end{array}$ & $\begin{array}{r}63,1 \\
36,4 \\
361,9 \\
41.200\end{array}$ & $\begin{array}{r}55,7^{\star \star} \\
32,0^{\star \star} \\
189,2^{\star \star \star} \\
36.097^{\star \star \star}\end{array}$ \\
\hline N (empresas) & 442 & 663 & 420 & 1.525 \\
\hline \% (empresas) & 29,0 & 43,5 & 27,5 & 100,0 \\
\hline
\end{tabular}

Fuente: Elaboración propia a partir de los datos de la ESEE. 
Sin embargo, sí se puede afirmar que la intensidad de uso de las tecnologías 14.0, entendida como un mayor número de usos de las cuatro tecnologías identificadas, se asocia a un proceso de generación de valor más intensivo en conocimiento y capital humano, más innovador, más digitalizado y más sostenible. Y, muy probablemente, en consonancia con esto, con unos resultados empresariales claramente más positivos (tabla 1). Si empezamos por el proceso de generación de valor, las empresas con unos usos más intensivos de las tecnologías 14.0 se caracterizan por un gasto en I+D (2,1 millones de euros de media), un porcentaje de empleados en actividades de I+D (12,3\%) y un gasto externo en formación por trabajador (139,2 euros) muy superiores a los otros dos niveles de uso. Del mismo modo, estas empresas también destacan por unos usos mucho más intensivos de la tecnología: presencia de una dirección o comité de tecnología (9,9\% del total de empresas), utilización de asesores tecnológicos (8,3\%) y evaluación de tecnologías alternativas (10,6\%). Las empresas más intensivas en la utilización de tecnologías 14.0 también destacan por una mayor propensión a la innovación: producto (6,1\%), proceso (13,9\%), organización $(8,1 \%)$ y comercialización $(6,2 \%)$, y por una mayor profundidad de los usos del comercio electrónico: compras digitales a los proveedores (14,4\%) y ventas digitales a las empresas (3,9\%). Por último, los usos más intensivos de las tecnologías 14.0 también se asocian a un proceso de generación de valor más limpio, puesto que también se obtiene una presencia significativamente más elevada que en los otros niveles de uso de la inversión $(10,6 \%)$ y del gasto $(21,0 \%)$ en protección medioambiental.

Probablemente, de acuerdo con este proceso de generación de valor más intensivo en tecnología, capital humano, innovación y digitalización, los resultados de las empresas más intensivas en los usos de las tecnologías 14.0 también son claramente mejores. En efecto, las empresas con usos tecnológicos 14.0 más intensivos se caracterizan por un volumen de ventas (150,8 millones de euros), un valor añadido (28,4 millones de euros) y unas exportaciones (91,7 millones de euros) que, al menos, duplican la media del conjunto de la empresa industrial. Asimismo, el margen bruto de explotación se sitúa más de dos puntos por encima de la media sectorial $7,2 \%$ frente al 5,1\%, respectivamente). Por último, también es reseñable que la empresa intensiva en tecnologías 14.0 es un 30\% más eficiente (productividad por trabajador o por hora trabajada) que las empresas que no usan estas tecnologías: 63,1 miles de euros por trabajador y 36,4 euros por hora trabajada. Del mismo modo, también es capaz de emplear a un número mucho mayor de trabajadores (361,9 empleados de media, prácticamente el doble de la media industrial) y de retribuirlos mucho mejor (41,2 miles de euros de coste laboral por trabajador, un $12,4 \%$ por encima de la media industrial).

\section{Conclusión: 14.0, ¿hacia una nueva base tecnológica de propósito general en la industria?}

Acabamos de constatar que unos mayores usos de las tecnologías 14.0 se asocian con un proceso de generación de valor de la empresa industrial más intensivo en I+D y en capital humano, más innovador y más sostenible. Probablemente, las relaciones de complementariedad establecidas entre la 14.0 y el resto de dimensiones tecnológicas y basadas en el conocimiento de las empresas determinen unos mejores resultados en términos de ventas, valor añadido, exportaciones, margen bruto de explotación, productividad, empleo y salarios. Sin embargo, el análisis realizado solo nos permite situar los resultados obtenidos en el terreno de la asociación estadística. Lamentablemente, la no disposición de datos sobre otras tecnologías 14.0 clave, como el big data, el cloud computing o el loT, y la falta de una serie temporal de datos nos ha impedido ir más allá. También es probable que en la explicación de la implantación y de los usos de estas tecnologías existan efectos de dimensión y de sector de actividad, de manera que estos también sean importantes en la determinación de los resultados empresariales derivados de la 14.0. Pero del análisis realizado sí podemos deducir que, a pesar de su estado incipiente, las tecnologías 14.0 manifiestan propiedades de plataforma tecnológica en el sentido de que se conectan con el resto de la base tecnológica e innovadora de las empresas. En la medida en que estas sean capaces de generar más relaciones de complementariedad con otros activos y dimensiones de la empresa, así como determinar nuevas fuentes de eficiencia, las tecnologías 14.0 podrían acabar consolidándose como una nueva base tecnológica de propósito general. 


\section{Bibliografía}

BODROZIC, A.; ADLER, P. S. (2018). «The Evolution of Management Models: A Neo-Schumpeterian Theory». Administrative Science Quarterly (vol. 63, núm. 1, pág. 85-129). https://doi.org/10.1177/0001839217704811

BRETTEL, M.; FRIEDERICHSEN, N.; KELLER, M.; ROSENBERG, M. (2014). «How Virtualization, Decentralization and Network Building Change the Manufacturing Landscape: An Industry 4.0 Perspective». International Journal of Mechanical, Industrial Science and Engineering (vol. 8, núm. 1, pág. 37-44).

DALENOGARE, L. S.; BENITEZ, G. B.; AYALA, N. F.; FRANK, A. G. (2018). «The expected contribution of Industry 4.0 technologies for industrial performance». International Journal of Production Economics (núm. 204, pág. 383-398). https://doi.org/10.1016/j.ijpe.2018.08.019

DE SOUSA-JABBOUR, A. B. L.; JABBOUR, C. J. C.; FOROPON, C.; GODINHO-FILHO, M. (2018). «When titans meet - Can industry 4.0 revolutionise the environmentally-sustainable manufacturing wave?». Technological Forecasting \& Social Change (núm. 132, pág. 18-25). https://doi.org/10.1016/j.techfore.2018.01.017

DÍAZ-CHAO, A.; SAINZ-GONZÁLEZ, J.; TORRENT-SELLENS, J. (2015). «ICT, innovation and firm productivity: New evidence from small local firms». Journal of Business Research (vol. 68, núm. 7, pág. 1439-1444). https://doi.org/10.1016/j.jbusres.2015.01.030

FRANK, A. G.; DALENOGARE, L. S.; AYALA, N. F. (2019). «Industry 4.0 technologies: Implementation patterns in manufacturing». International Journal of Production Economics (núm. 210, pág. 15-26). https://doi.org/10.1016/j.jpe.2019.01.004

JESCHKE S.; BRECHER C.; MEISEN, T.; ÖZDEMIR, D.; ESCHERT T. (2017). «Industrial Internet of Things and Cyber Manufacturing Systems». En: JESCHKE, S.; BRECHER, C.; SONG, H.; RAWAT, D. (eds.). Industrial Internet of Things. Cham: Springer. https://doi.org/10.1007/978-3-319-42559-7

KAGERMANN, H.; WAHLSTER, W.; HELBIG, J. (2013). Recommendations for implementing the strategic initiative Industrie 4.0: Securing the future of German manufacturing industry. Final report of the Industrie 4.0 Working Group. Acatech: Forschungsunion.

LONGO, F.; NICOLETTI, L.; PADOVANO, A. (2017). «Smart operators in industry 4.0: A human-centered approach to enhance operators' capabilities and competencies within the new smart factory context». Computers \& Industrial Engineering (núm. 113, pág. 144-159). https://doi.org/10.1016/j.cie.2017.09.016

LU, Y. (2017). «Industry 4.0: A survey on technologies, applications and open research issues». Journal of Industrial Information Integration (vol. 6, pág. 1-10). https://doi.org/10.1016/j.jii.2017.04.005

MONOSTORI, L.; KÁDÁR, B.; BAUERNHANSL, T.; KONDOH, S.; KUMARA, S.; REINHART, G.; SAUER, O.; SCHUH, G.; SIHN, W.; UEDA, K. (2016). "Cyber-physical systems in manufacturing". CIRP Annals Manufacturing Technology (vol. 65, núm. 2, pág. 621-641).

MÜLLER, O.; FAY, M.; VOM BROCKE, J. (2018). «The Effect of Big Data and Analytics on Firm Performance: An Econometric Analysis Considering Industry Characteristics". Journal of Management Information Systems (vol. 35, núm. 2, pág. 488-509). https://doi.org/10.1080/07421222.2018.1451955

PORTER, M. E.; HEPPELMANN, J. E. (2014). «How Smart, Connected Products Are Transforming Competition». Harvard Business Review (vol. 92, núm. 11, pág. 64-88).

PORTER, M. E.; HEPPELMANN, J. E. (2015). «How Smart, Connected Products Are Transforming Companies». Harvard Business Review (vol. 93, núm. 10, pág. 96-114).

SCHUH, G.; ANDERL, R.; GAUSEMEIER, J.; TEN HOMPEL, M.; WAHLSTER, W. (2017). Industrie 4.0 Maturity Index. Managing the Digital Transformation of Companies. Munich: Acatech.

TAO, F.; CHENG, J.; QI, Q.; ZHANG, M.; ZHANG, H.; SUI, F. (2018). «Digital twin-driven product design, manufacturing and service with big data». International Journal of Advanced Manufacturing Technology (vol. 9, núm. 9-12, pág. 3563-3576). https://doi.org/10.1007/s00170-017-0233-1

TORRENT-SELLENS, J. (2004). Innovació tecnològica, creixement econòmic i economia del coneixement. BarceIona: Consell de Treball, Econòmic i Social de Catalunya (CTESC), Generalitat de Catalunya. 
TORRENT-SELLENS, J. (2018). Las empresas industriales en 2016. Encuesta sobre Estrategias Empresariales (ESEE). Robótica, productividad y empleo en la empresa industrial. Madrid: Ministerio de Hacienda, Gobierno de España.

TORRENT-SELLENS, J.; DÍAZ-CHAO, A. (2018). «Coneixement, robòtica i productivitat a la PIME industrial catalana: evidència empírica multidimensional». En: FERRÀS, X.; ALCOBA, O.; TORRENT-SELLENS, J. (coords.). Transformació digital i intel/ligència artificial. Barcelona: Col.legi d’Economistes de Catalunya (pág. 91-126).

TRAJTENBERG, M. (2018). «Al as the next GPT: A Political-Economy Perspective». National Bureau of Economic Research (NBER) Working Paper (núm. 24245). https://doi.org/10.3386/w24245

WANG, S.; WAN, J.; LI, D.; ZHANG, C. (2016). «Implementing Smart Factory of Industrie 4.0: An Outlook». International Journal of Distributed Sensor Networks (vol. 12, núm. 1, pág. 1-10). https://doi.org/10.1155/2016/3159805

WEI, Z.; SONG, X.; WANG, D. (2017). «Manufacturing flexibility business model design, and firm performance». International Journal of Production Economics (núm. 193, pág. 87-97). https://doi.org/10.1016/j.jppe.2017.07.004

WELLER, C.; KLEER, R.; PILLER, F. T. (2015). «Economic implications of 3D printing: Market structure models in light of additive manufacturing revisited». International Journal of Production Economics (núm. 164, pág. 4356). https://doi.org/10.1016/j.jpe.2015.02.020

XU, L. D.; XU, E. L.; LI, L. (2018). «Industry 4.0: state of the art and future trends». International Journal of Production Research (vol. 56, núm. 8, pág. 2941-2962). https://doi.org/10.1080/00207543.2018.1444806

ZHONG, R. Y.; XU, X.; KLOTZ, E.; NEWMAN, S. T. (2017). «Intelligent Manufacturing in the Context of Industry 4.0: A Review». Engineering (vol. 3, núm. 5, pág. 616-630). https://doi.org/10.1016/J.ENG.2017.05.015

Cita recomendada: TORRENT-SELLENS, Joan. Industria 4.0 y resultados empresariales en España: un primer escaneado. Oikonomics [en línea]. Noviembre 2019, no. 12, pp. 1-11. ISSN: 23399546. DOI: https://doi.org/10.7238/o.n12.1910

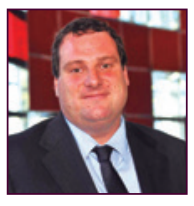

Joan Torrent-Sellens

jtorrent@uoc.edu

Estudios de Economía y Empresa, Universitat Oberta de Catalunya (UOC)

Catedrático de Economía de los Estudios de Economía y Empresa de la Universitat Oberta de Catalunya (UOC). Director del grupo interdisciplinar de investigación sobre las TIC, i2TIC (http://i2TIC.net). Especialista en el análisis económico de la transformación digital y de la economía del conocimiento, temática sobre la que ha publicado cuarenta libros y capítulos de libro, y noventa artículos en revistas de investigación indexadas.

Los textos publicados en esta revista están sujetos -si no se indica lo contrario- a una licencia de Reconocimiento 4.0 Internacional de Creative Commons. Puede copiarlos, distribuirlos, comunicarlos públicamente, hacer obras derivadas siempre que reconozca los créditos de las obras (autoría, nombre de la revista, institución editora) de la manera especificada por los autores o por la revista. La licencia completa se puede consultar en https://creativecommons.org/licenses/by/4.0/deed.es_ES.

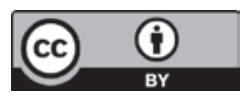

